fournal of Medical Genetics (1976). 13, 237-251.

\section{Partial trisomy 9 with resemblance to Coffin-Siris syndrome*}

Summary. An infant with partial trisomy of chromosome 9 who phenotypically resembled a case of Coffin-Siris syndrome is presented.

In the recent published reports, two cases of trisomy 9 have been reported. One of these was a complete trisomy of chromosome 9 (Feingold and Atkins, 1973) and the other a trisomy 9 mosaicism (Haslam et al, 1973). Our patient had a karyotype of $47, X X,+9 q-$; to the best of our knowledge no such patient has been previously reported.

* Supported in part by National Foundation-March of Dimes Grant C152.

\section{Case report}

A Black baby girl was born of an 18-year-old gravida 1, para. 1, abortion 0 Black female and a 22-year-old Black male, with normal maternal and paternal pedigrees. Pregnancy and labour were uneventful, and the infant was delivered by low outlet forceps. At 1 minute the Apgar score was 6, and at 5 minutes it was 7 . At birth, the infant weighed $2545 \mathrm{~g}$; birth length was $46 \mathrm{~cm}$; and head circumference was $34 \mathrm{~cm}$. Her face revealed moderate micrognathia, a depressed nasal bridge, bulbous nose tip, and thick lips (Fig. 1); her ears were $30^{\circ}$ posteriorly rotated with abnormal antihelices and antitragi and a preauricular dimple on the left side (Fig. 2). Enophthalmos was noted especially on the right side. Examination of the extremities showed shortening and clinodactyly with hypoplastic fifth nails bilaterally; there was generalized mild shortening of the remaining digits, with mild degrees of nail hypoplasia (Fig. 3). A simian palmar crease was noted on the right hand. She also had mild hirsutism over the scapular and lower back areas.

The infant remained in the Martland Hospital Unit Intensive Care Nursery because of failure to thrive. During her stay in the nursery she was found to be anaemic and developed an omphalitis for which she was

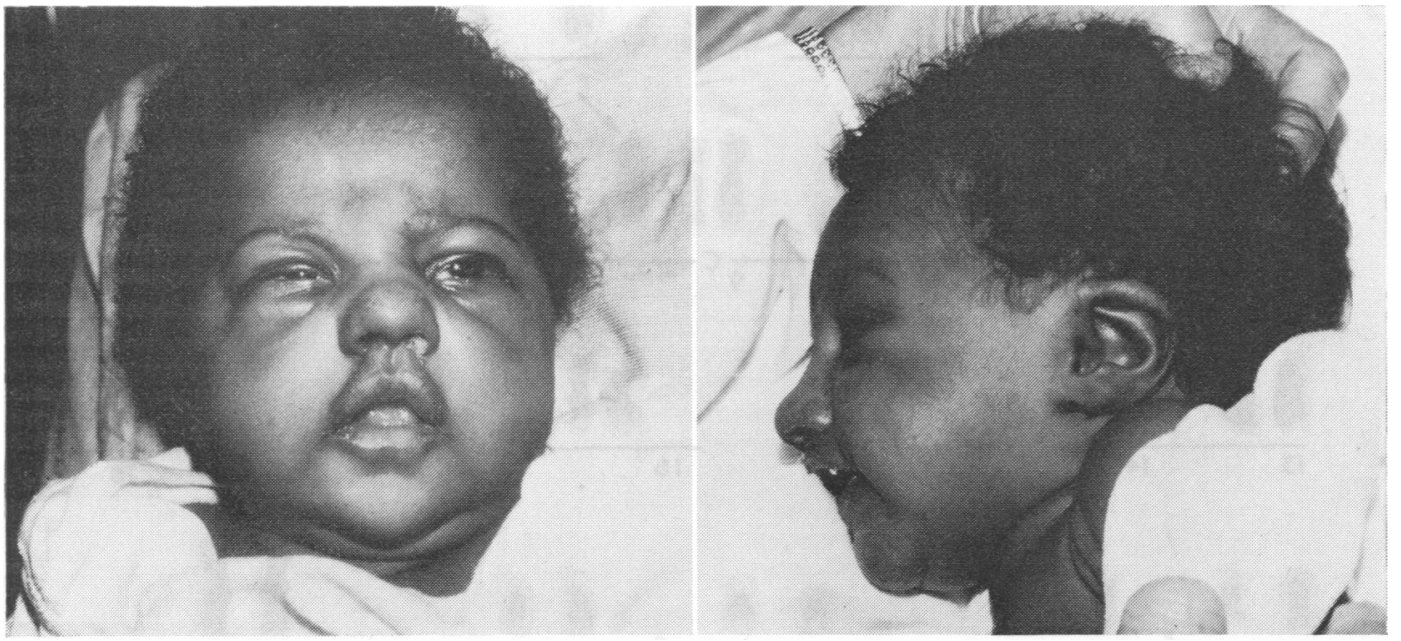

Fig. 1. Patient's facies showing the bulbous nose, enophthalmos, and thick lips. 


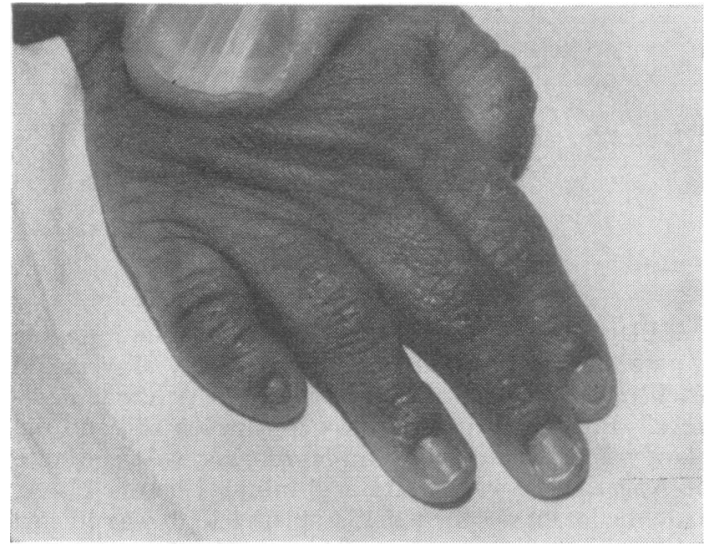

FIG. 3. Patient's hand with short fifth finger and nail hypoplasia; and mild hypoplasia of the other nails.

treated with antibiotics. She was discharged at the age of 3 weeks.

At 4 weeks, the infant was readmitted with bronchopneumonia. At this time a grade $3 / 6$ systolic ejection murmur was detected over the fourth left intercostal space and pulmonary area; she subsequently developed congestive heart failure which required digoxin therapy. Cardiac catheterization studies revealed a ventricular septal defect and a persistent ductus arteriosus with pulmonary hypertension. The infant was discharged at 2 months of age and did well, until she was readmitted with septicaemia and meningitis at age 4 months; she died several days later. Necropsy revealed a purulent meningitis and atrial and ventricular septal defects. No other major gross abnormalities were found.

\section{Cytogenetic investigation}

Routine chromosomal analysis showed 47 chromosomes in each of 60 cells counted. The extra chromosome morphologically resembled the No. 9 chromosome. Giemsa banding $\dagger$ revealed that the extra chromosome was a No. 9 with partial long arm deletion: a karyotype of $47, \mathrm{XX},+9 \mathrm{q}-$ (Fig. 4). Chromosomal analysis performed on the mother was normal. We could not obtain the father's chromosomes.

\section{Discussion}

When first examined, the initial diagnostic impression on the baby was the Coffin-Siris syndrome. In this syndrome, the major features, as reported by Weiswasser et al (1972), include a wide bulbous nose, low nasal bridge, moderate hirsutism, and

† Bio Science Labs, Van Nuys, California.
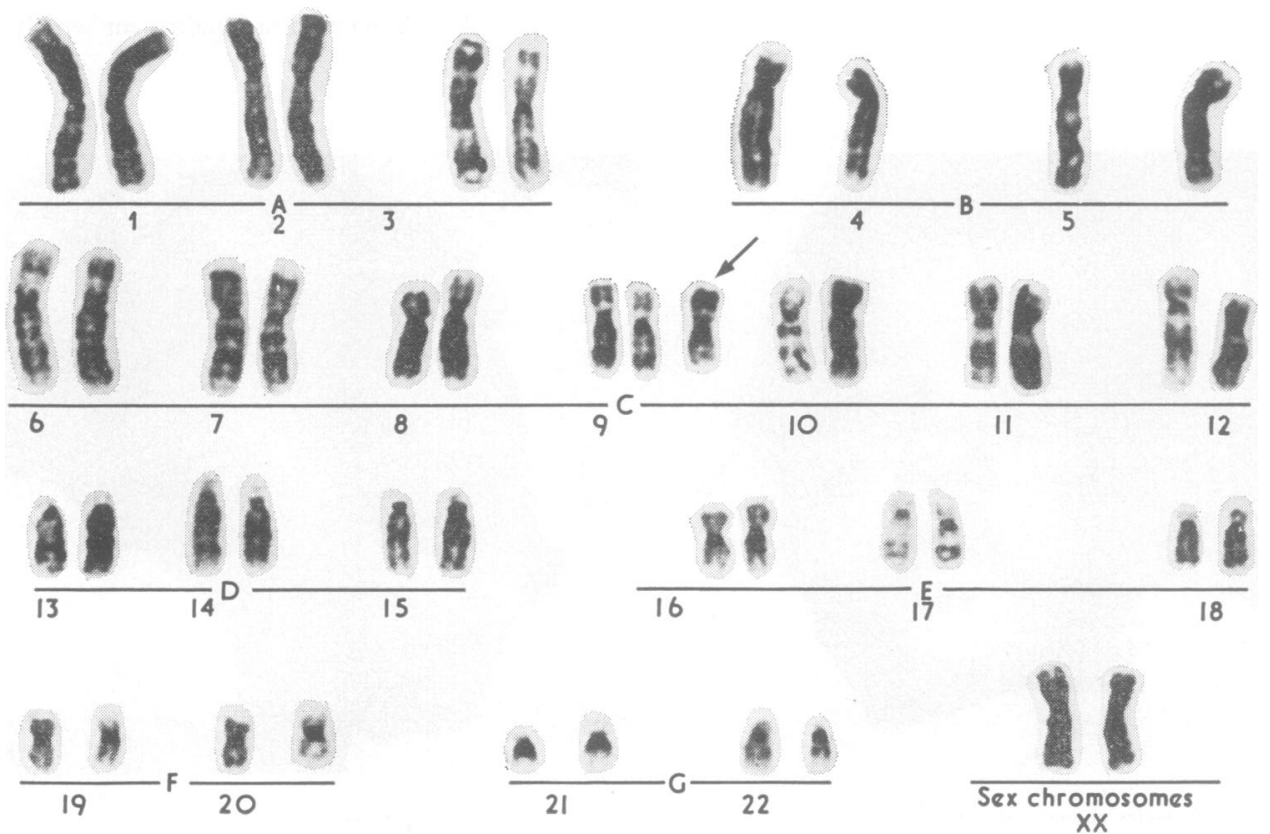

FIG. 4. Karyotype with Giemsa banding revealing pattern of $47, X X,+9 q-$. 
digital anomalies with nail hypoplasia especially of the fifth fingers. Our patient did have all of these characteristics. The patients described as having Coffin-Siris syndrome have had no demonstrable chromosomal abnormalities; yet, our patient did have an abnormal karyotype of $47, \mathrm{XX},+9 \mathrm{q}-$. The significance of the resemblance of our patient to those with the Coffin-Siris syndrome obviously cannot be determined until more cases with both types of abnormalities have been reported.

As previously noted, a patient with trisomy 9 was reported by Feingold and Atkins (1973). Their patient survived 28 days and showed microcephaly, low set malformed ears, enophthalmos, a bulbous nose, severe micrognathia, low hairline, heart defects, missing phalanges from toes two to five, abnormal external genitalia, and deformities of the central nervous system. In 1973, Haslam et al described a 9-year-old boy with severe mental retardation, short stature, hypotonia, heart defects, genital anomalies, and brain abnormalities. Genotypically he was found to be a trisomy 9 mosaic. Others (Rethoré et al, 1970; Hoehn, Engel, and Reinwein, 1971) have reported patients with trisomy of the short arm of chromosome 9. Collectively these patients revealed severe mental retardation, enophthalmos, mild hypertelorism, bulbous noses, and anomalies of the ear and phalanges. Our patient does indeed resemble these patients, having many of their features; yet, her chromosomal pattern is different. In fact, this is probably the first reported case of trisomy 9 with partial deletion of its long arm.

It is of great interest that our patient resembled those with the Coffin-Siris syndrome as well as those with trisomies for chromosome 9. Again the relevance of these relationships must await further case reports before any speculations can be made.

\section{Theodore Kushnick and Giacomo M. Adessa}

The Division of Human Genetics Department of Pediatrics, New Fersey Medical School, Newark, New Fersey, $U S A$

REFERENCES

Feingold, M. and Atkins, L. (1973). A case of trisomy 9. Fournal of Medical Genetics, 12, 184-187.

Haslam, R. H., Broske, S. P., Moore, C. M., Thomas, G. H., and Neill, C. A. (1973). Trisomy 9 mosaicism with multiple congenital anomalies. Fournal of Medical Genetics, 10, 180-184.

Hoehn, H., Engel, W., and Reinwein, H. (1971). Presumed trisomy for short arm of chromosome no. 9 not due to inherited translocation. Humangenetik, 12, 175-181.
Rethoré, M., Larget-Piet, L., Abonyi, D., Boeswillwald, M., Berger R., Carpentier, S., Cruveiller, J., Dutrillaux, B., Lafourcade, J., Penneau, M., and Lejeune, J. (1970). Sur quatre cases de trisomie pour le bras court du chromosome 9. Individualisation d'une nouvelle entité morbide. Annales de Génétique, 13, 217-232.

Weiswasser, W. H., Hall, B. O., Delavan, G. W., and Smith, D. W. (1972). Coffin-Siris syndrome. Two new cases. American fournal of Diseases of Children, 125, 838-840.

\section{Pure partial trisomy for long arm of chromosome 9}

Summary. A case of a 4-year-old boy with trisomy of the long arm of chromosome 9 is described $(46, \mathrm{XY}, \operatorname{der}(9)$, $t(9 ; 9)(q 32 ; q 12))$. The trisomy is probably the result of a translocation of the long arm of the chromosome from one homologue to the other in a parental gonad. The clinical features of the child which include severe developmental retardation, bird-like facies, tapered fingers, and flexion contractures of the legs are similar to those of the few cases described of trisomy of the whole chromosome.

Trisomy 9 has been reported very rarely in living individuals. It has been found in all the cells examined in a newborn child with multiple congenital abnormalities (Feingold and Atkins, 1973) and also in a proportion of the cells of a newborn infant (Bowen, Ying, and Chung, 1974) and a child of 9 years (Haslam et al, 1973) both of whom had severe congenital abnormalities.

We wish to report a boy with multiple congenital abnormalities and severe developmental retardation in whom all cells examined contained an abnormal chromosome 9 which had a substantial portion of the long arm (q12 $\rightarrow \mathrm{q} 32)$ present in duplicate. As only chromosome 9 was involved this was a case of pure trisomy for this segment of chromosome. We are not aware of any previous reports of partial trisomy for the long arm of chromosome 9.

\section{Case report}

This child, a boy of birthweight $1730 \mathrm{~g}$, was born by spontaneous vertex delivery at 36 weeks' gestation after an uneventful pregnancy. Incubator care was required until 2 months of age; he was discharged at 3 months weighing $2250 \mathrm{~g}$. Subsequent clinic attendances showed developmental milestones to be greatly delayed. 